\title{
Visible Light Communications: Fast-Orthogonal Frequency Division Multiplexing in Highly Bandlimited Conditions
}

\author{
Paul Anthony Haigh and Izzat Darwazeh
}

\begin{abstract}
In this paper we propose, for the first time, fast orthogonal frequency division multiplexing (FOFDM) for visible light communications (VLC) systems. VLC systems often exhibit highly band-limited system responses and as such supporting high transmission speeds is a key challenge. FOFDM makes use of an inverse discrete cosine transform (IDCT) to generate the time domain symbols, as opposed to the inverse fast Fourier transform (IFFT) used in OFDM. This offers several advantages for VLC in particular, because sacrificing complex modulation formats in favour of real ones such as pulse-amplitude modulation (PAM) enables reduction of the subcarrier spacing to $1 / 2 T$, where $T$ is the symbol period, whilst maintaining orthogonality. This results in a bandwidth saving of $50 \%$ in comparison to OFDM, whilst maintaining an equivalent spectral efficiency. Hence in this work, we examine the bit error rate performance of F-OFDM in comparison to conventional OFDM with an equal number of subcarriers and equivalent spectral efficiency for a number of band-limited conditions as a function of the energy-per-bit to noise spectral density ratio $\left(E_{b} / N_{0}\right)$. We demonstrate that due to the $50 \%$ bandwidth savings, F-OFDM outperforms OFDM in band-limited conditions, because the impact of the attenuation caused by the band-limitation on each subcarrier is reduced. Therefore, we show that FOFDM results in lower electrical power penalty relative to conventional OFDM for a given set of band-limitation conditions, while maintaining equivalent spectral efficiency.
\end{abstract}

Index Terms-Digital signal processing, modulation formats, optical communications, orthogonal frequency division multiplexing, visible light communications

\section{INTRODUCTION}

Visible light communications (VLC) is an emerging access network technology that has become the focus of enormous attention in recent years. Leveraging on existing light-emitting diode (LED) infrastructure, VLC is capable of simultaneously offering communications and illuminations, typically, but not exclusively, within an indoor environment. One of the major advantages of VLC over traditional radio networks is the $\sim 300 \mathrm{THz}$ license free bandwidth that is available in the visible region; approximately $\sim 10,000$ times larger than the radio frequency range. Considering that there is an exponentially increasing end-user demand for data, which is constantly outstripping supply [1], VLC is a promising lowcost and complementary alternative to radio-based wireless communications systems.

Authors are with the Communications and Information Systems Group, Department of Electrical and Electronic Engineering, University College London, London, WC1E 6BT, UK

e-mail: \{p.haigh; i.darwazeh\}@ucl.ac.uk
One of the main research challenges in VLC has emerged from the LED devices themselves, which are typically either gallium nitride (blue emitting) chips with a cerium doped yttrium aluminium garnet (yellow-ish) phosphor colour converter, or individual red, green and blue (RGB) chips packaged into a single device [2]. Using RGB-LEDs introduces a colour balancing problem, although $\mathrm{Gb} / \mathrm{s}$ transmission speeds have been reported [2]. On the other hand, using a phosphor LED simplifies the colour conversion problem but inhibits high transmission speeds because of the limited transient response of the phosphor. To overcome this challenge, researchers have employed high spectral efficiency modulation such as orthogonal frequency division multiplexing (OFDM) [3] and (multiband) carrier-less amplitude and phase modulation (CAP) [4]. Both of these modulation formats have demonstrated considerable success in achieving high transmission speeds as $\sim 3 \mathrm{~Gb} / \mathrm{s}$ were demonstrated for both formats using the same physical link in [2].

A new paradigm has recently emerged in VLC that makes use of organic light-emitting diodes (OLEDs) instead of the more conventional inorganic devices mentioned above. OLEDs can be loosely grouped into two categories: $(i)$ small-molecule OLEDs (SMOLEDs) [5] and (ii) polymer LEDs (PLEDs) [6]. SMOLEDs are normally processed via evaporation in highvacuum chambers, resulting in brittle crystals as can be found in inorganic LEDs. A more suitable and attractive option is offered by PLEDs, which can be easily processed using a number of solution-based methods [7]. This is advantageous as wet methods result in ultra-low-cost thin film screens of individually addressable pixel matrices for applications such as VLC-enabled integrated smart device monitors. There have been reports in the literature of PLEDs with exceptionally small photo-active areas (several $\mu \mathrm{m}^{2}$ ) that offer bandwidths in excess of $60 \mathrm{MHz}$ [8], however, typical bandwidths reported in the literature are in the region of several hundred $\mathrm{kHz}$. For instance [9] reports three PLEDs with different emissive layer materials demonstrating bandwidths ranging from 110$600 \mathrm{kHz}$. Therefore, in order to provide an integrated solution into a high speed VLC access network, it is necessary to increase further the available transmission speeds by introducing more advanced modulation schemes.

As a result of such low bandwidths, it is desirable to investigate the feasibility of other advanced modulation formats in a band-limited environment. In this work we propose, for the first time, the use of fast-OFDM (F-OFDM) for VLC, where real data symbols are used to modulate subcarriers 
using the inverse discrete cosine transform (IDCT). In FOFDM, these subcarriers are spaced at $1 / 2 T$ intervals, where $T$ is the symbol period, whilst maintaining orthogonality, in contrast to OFDM where $1 / T$ subcarrier spacing is employed. In F-OFDM, orthogonality is maintained by restricting the modulation format to real-valued 1-dimensional modulation formats [10]. F-OFDM was originally proposed for wireless systems, however, it has found application in optical fibre systems as reported in [11-14] and is a good candidate for VLC because the inverse discrete cosine transform (IDCT) output is real-valued, as is required for a non-coherent intensity modulated system such as VLC. This means that one can avoid the use of Hermitian symmetry, which is required in conventional OFDM systems, while using half the bandwidth, which is advantageous particularly for the low bandwidth polymer photonic devices that we propose to use. In this work, we investigate, by numerical simulations, the comparative bit error rate (BER) performance of F-OFDM and OFDM in a highly band-limited VLC system based on a PLED device used in our previous work [9]. To evaluate the performance of both formats in a band-limited environment, we set the signal bandwidth in excess of the fixed PLED bandwidth to induce inter-symbol interference (ISI), as will be explained in the next section. Furthermore, we vary the noise power present in the system to investigate the BER profiles of each modulation format. We compare the performance of two systems operating at the same bit rate, but with different modulation formats; an OFDM system and an FOFDM one where the FOFDM signals occupy half the bandwidth, and therefore both systems have the same spectral efficiency [10]. As the FOFDM system saves bandwidth in the band-limited channels tested, it offers improved bit error rate (BER) performance in comparison to OFDM, even when the signal bandwidth exceeds the modulation bandwidth by a factor of ten. We verify through a set of system studies that the attenuation experienced on the higher frequency subcarriers from the band-limitation is less in F-OFDM than in OFDM.

The paper is organised as follows. In Section II, the test setup and modulation formats are described. In section III, the results are shown and in Section IV, conclusions are drawn.

\section{Test Setup}

A schematic block diagram of the systems under test is illustrated in Fig. 1. A $2^{23}-1$ pseudo-random binary sequence is generated to represent the bitstream $d$. Since the IDCT is used to generate the time domain samples of the F-OFDM signal (i.e. no imaginary components), while an OFDM signal is generated using an inverse fast Fourier transform (IFFT), it is necessary to use different modulation schemes that offer different constellation dimensionality, to maintain equal spectral efficiencies for fair comparison of the systems. For FOFDM, multi-level pulse amplitude modulation is employed, which is a 1-dimensional constellation and termed in this paper as $M$-ary, while the 2-dimensional quadrature amplitude modulation (QAM) is used for OFDM, and referred to in this work as $M_{o}$-ary. The symbols $c$ are passed through a serialto-parallel converter, which distributes the symbols into either

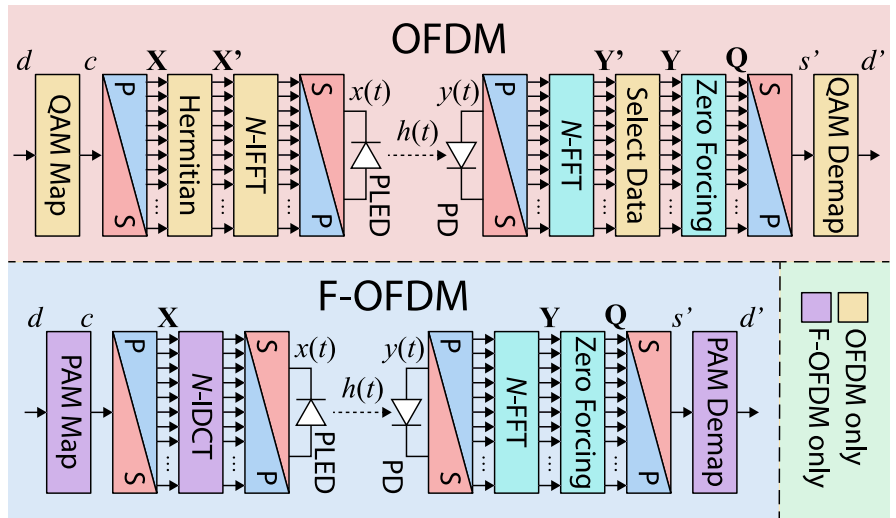

Fig. 1. The schematic block diagram for the system under test; the conventional OFDM system is shown at the top of the figure, and the proposed F-OFDM figure at the bottom. There are several differences between the two formats, such as the fact that PAM and an IDCT is used for F-OFDM, while QAM and an IFFT are used for OFDM. These are highlighted as seen on the right.

an $N$-element parallel vector for FOFDM or conventional OFDM modulation, respectively, which we denote as $\mathbf{X}$, and the number of subcarriers are given by $N$.

In intensity modulated OFDM-based VLC systems, generally the literature has reported the use of Hermitian symmetry in order to obtain a real-valued output from the IFFT. This means that half of the subcarriers must be modulated with the conjugate of the corresponding symbol, i.e. $c_{-n}=c_{n}^{*}$ for the $n^{\text {th }}$ subcarrier and $n \in[-N / 2, \ldots, N / 2-1]$.

The more common IFFT is given by the following [15]:

$$
x(m)=\frac{1}{\sqrt{N}} \sum_{n=0}^{N-1} c_{n} \exp \left(\frac{j 2 \pi m n}{N}\right)
$$

where $x(m)$ is the $m^{\text {th }}$ time sample of one OFDM symbol and $c_{n}$ is the symbol modulated on the $n^{\text {th }}$ subcarrier. Similarly, the IDCT is given by [16]:

$$
x(m)=\sum_{n=0}^{N-1} w_{n} c_{n} \cos \left(\frac{\pi m n}{N}\right)
$$

where $w_{n}$ is a normalisation factor given by:

$$
w_{n}=\left\{\begin{array}{l}
\frac{1}{\sqrt{N}}, n=1 \\
\sqrt{\frac{2}{N}}, 2 \leq n \leq N
\end{array}\right.
$$

Crucially, the subcarrier spacing $\Delta f$ for F-OFDM and OFDM are not equal, as is reflected in eqs. (1) and (2). For OFDM, it is well known that $\Delta f=\frac{r_{s}}{N}$, while for F-OFDM, $\Delta f=\frac{r_{s}}{2 N}$, where $r_{s}$ is the symbol rate [10], whilst maintaining orthogonality, the mathematical proof of which can be found in [16]. This concept is illustrated in Fig. 2. It should be noted that there is an excess bandwidth factor associated with both FOFDM and OFDM [17], which tends to zero as $N$ tends to infinity. Therefore, for a large enough $N$, this allows double the amount of subcarriers to be employed for FOFDM in comparison to OFDM over an equivalent signal bandwidth. For wideband channels, where the channel bandwidth is wider than the signal bandwidth, the approach of doubling the number of subcarriers should 


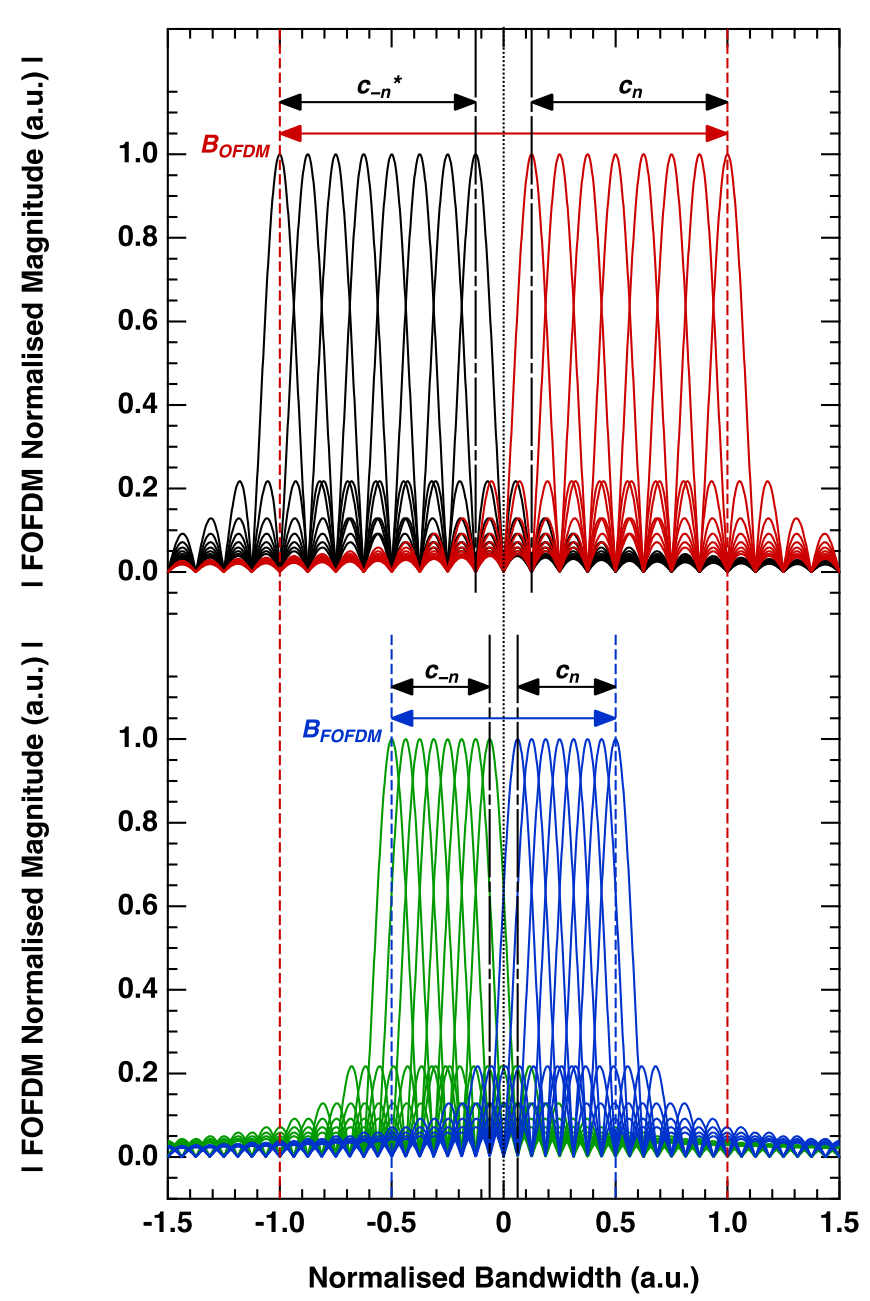

Fig. 2. The subcarrier arrangement for F-OFDM (top) and OFDM (bottom), it is noteworthy that F-OFDM is capable of supporting twice as many subcarriers as OFDM, whilst maintaining orthogonality, by restricting the modulation format to real 1-dimensional formats, thus allowing subcarriers to be spaced at $1 / 2 T$, half the spacing required for OFDM at $1 / T$.

be followed to increase spectral efficiency. However, in bandlimited channels, such as those employing PLEDs, we aim to reduce bandwidth and therefore set the number of subcarriers as $N$, for both F-OFDM and OFDM, which will maintain the data rate but reduce the bandwidth requirement to half. We believe this is an advantageous approach for polymerbased communications systems and VLC in general because the band-limitation is the prohibitive factor towards supporting high transmission speeds. We postulate that using smaller subcarrier bandwidths in an attenuating environment results in closer approximation to a flat-band response-per-subcarrier and hence higher SNR-per-subcarrier. This motivation was proved in $[18,19]$, which shows as the number of subcarriers in a highly band-limited non-orthogonal multi-band system increases, the SNR/subcarrier increases, thereby enabling higher transmission speeds to be supported. This work is based on the multi-band CAP modulation, and the main disadvantages, without detailing the system operation, are two-fold: $(i)$, the sub-bands are not orthogonal and hence bandwidth is not utilised as efficiently as possible, and, (ii) the system requires numerous high order finite impulse response (FIR) filters which, when realised digitally, are computationally expensive

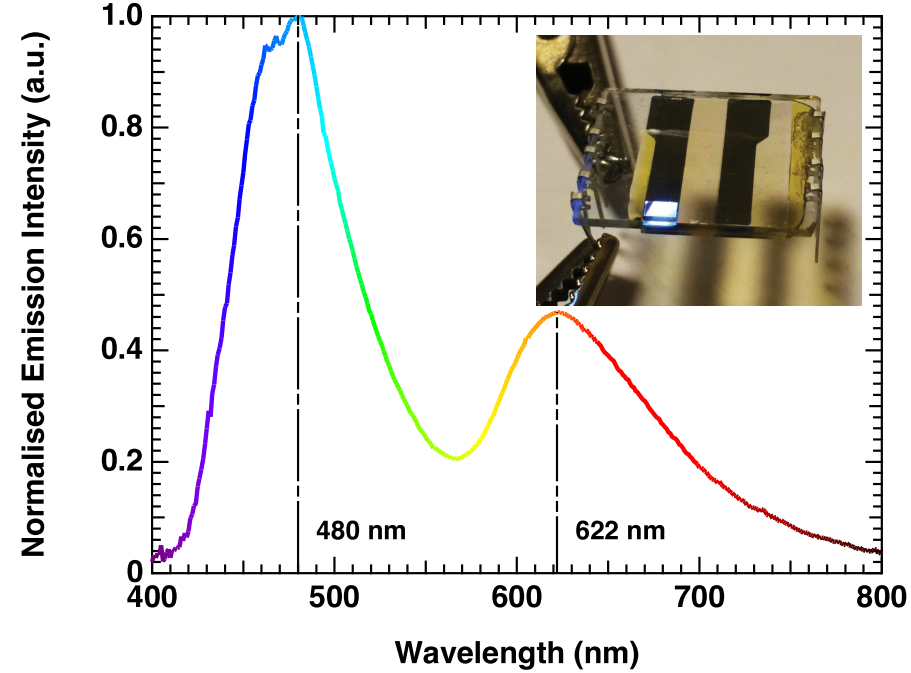

Fig. 3. The measured and normalised emission intensity of the PLED under test. The spectrum was captured using a calibrated photodetector, details can be referred to in [9].

in comparison to a IDCT or IFFT.

The range of $N$ tested in this work is $N=2^{4: 10}$. The number of bits-per-symbol for the FOFDM format $k=[1,2]$. In order to make like-for-like comparisons, the spectral efficiencies of both systems are kept equal and hence, the number of bits-per-symbol for OFDM, $k_{o}=2 k$. The modulation cardinality is given by $M=2^{k}$.

In both systems, the time samples obtained from either the IFFT or the IDCT are used, after parallel-to-serial conversion to intensity modulate a PLED. The models used for the PLED in this work are obtained from detailed characterisation carried out on real devices, where specific details of the PLED processing procedure can be found in [9]. It is worth noting that the emissive layer of the device was a blend of three polymers (F8:TFB:PFB) in a 1:2:2 ratio $[6,9,20,21]$, which approaches white-light emission as can be seen inset in Fig. 3, which also shows the normalised emission spectrum with its peak around the $\sim 480 \mathrm{~nm}$ (blue) and a tail that peaks at $\sim 622 \mathrm{~nm}$ (yellow-orange-red).

The L-I-V curve and bandwidth measurements are shown in Fig. 4 and the details of the measurement techniques can be found in [9]. An important characteristic demonstrated by the PLED modelled here is that the luminance-current (L-I) relationships exhibit high linearity. This is important because it ensures protection against clipping and distortion for modulation formats such as OFDM and F-OFDM, which often feature a high peak-to-average power ratio (PAPR). The PAPR is given by $[3,22]$ :

$$
P A P R=10 \log _{10}\left[\frac{\max _{0 \leq t \leq T}|x(t)|^{2}}{E\left[|x(t)|^{2}\right]}\right]
$$

The PLED bandwidth $f_{c}$ was measured at $\sim 600 \mathrm{kHz}$ using an Agilent EXA N9010A electrical spectrum analyser in [9]. The PLED demonstrates a first order low-pass filter response following the resistor-capacitor model, as reported for many 
PLED links in the literature $[9,23]$ as follows:

$$
H_{s}(f)=\frac{1}{\sqrt{1+\left(\frac{f}{f_{c}}\right)^{2}}}
$$

where $f$ is the frequency range of the signal. The intensity modulated signal is then transmitted over a static indoor channel, whose gain $H(0)$ is given by the expression [24]:

$$
H(0)=\frac{A\left(m_{l}+1\right)}{d_{r}^{2}} \cos (\theta) \cos (\varphi)
$$

where $A$ is the photoactive area of the receiver, $m_{l}$ is the Lambertian order of the emitter, $m_{l}=1$ for the PLED under test, $d_{r}$ is the transmitter-receiver distance, $\theta$ is the angle of light emission and $\varphi$ is the angle of incidence onto the photodiode. The line-of-sight configuration is used in this work and hence $\theta=\varphi=0$ to ensure maximum power transfer. By inspection of eq. (5), it is clear that with fixed $A$, and $\theta=\varphi=0$, as is often typical in experimental VLC systems $[4,15,25,26]$, the received power intensity is only distance dependent $\left(\propto d_{r}^{2}\right)$. In VLC systems, the electrical noise generated at the receiver is the dominant noise source and can be modelled as additive white Gaussian noise (AWGN). Therefore, in this work, we model the channel and noise simultaneously by varying the noise power whilst keeping a constant received signal power intensity to control the energy-per-bit to noise spectral density $\left(E_{b} / N_{0}\right)$ ratio over the range $0-40 \mathrm{~dB}$.

After transmission over the channel, the signal is detected by a photodiode (PD) which is modelled with ideal response, since commercially available silicon photodiodes with inbuilt transimpedance amplifiers have bandwidths substantially beyond that of the PLED device used here. Hence, if coupled with a properly designed low-pass output filter with cut-off frequency matched to the signal bandwidth, the PD will cause no limitations to the link performance. After photodetection, the signals are then passed through an $N$-point serial-toparallel converter and both formats are transformed by an FFT. It should be noted that at the receiver, the only difference between using a DCT and an FFT for F-OFDM is an issue of complexity. A DCT offers lower complexity due to implementation of a similar number of multiplications and additions, however, only for the in-phase component, since there is no quadrature part. In this work, which does not consider computational complexity in detail, we use an FFT for the FOFDM transform because it highlights the fact that in-phase orthogonality is maintained while the quadrature component experiences interference due to imaginary components generated by the receiver FFT, which are non-orthogonal. This issue is explored in detail in [10] and will become evident upon inspection of the constellations in the results section.

Next, for an OFDM system, the data carried on the conjugate subcarriers is discarded. For both systems, the data is equalised using a one-tap zero forcing equaliser, as is conventional in the VLC literature [27], to estimate and attempt to compensate for the combined PLED low-pass and channel responses. The symbols are then de-mapped from their constellations to give hard estimates of the transmitted data and for bit-by-bit BER measurement.

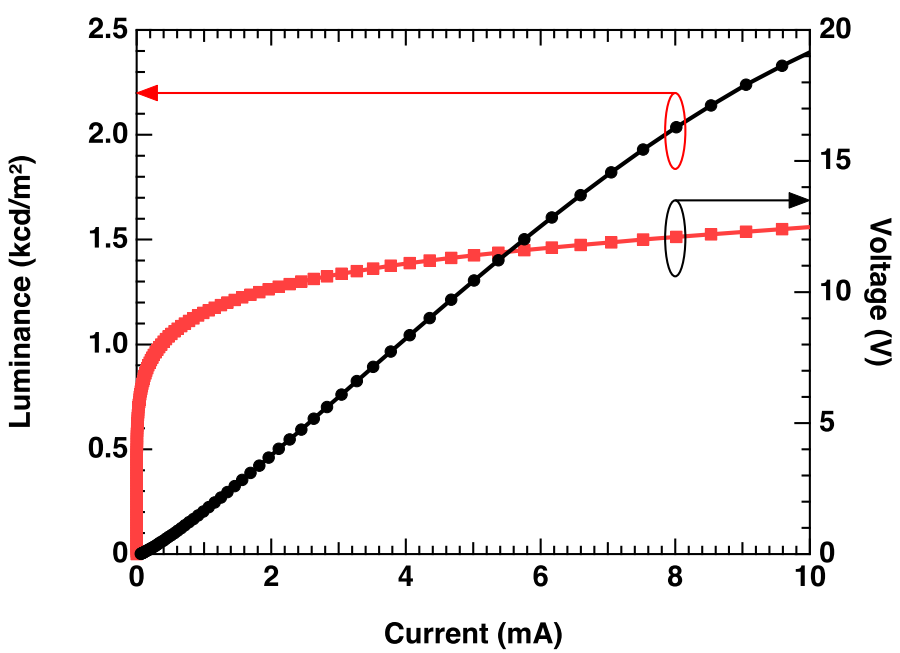

Fig. 4. The measured luminance-current and current-voltage (L-I-V) relationships for the PLED under test. The device exhibits strong linearity in its L-I relationship which is advantageous to avoid clipping of peaks. For details of the measurement used, refer to [9].

In this work, we investigate the performance of $\mathrm{F}$ OFDM in comparison to a conventional OFDM link under various band-limited conditions. The PLED bandwidth $f_{c}$ is fixed at $600 \mathrm{kHz}$ according to the measured bandwidth, while the signal bandwidths are set variably according to $f_{c}$. It should be noted that signal bandwidth $B$ is different for each modulation format, where OFDM has $B_{O F D M}$ signal bandwidth and $B_{F O F D M}$ is half of that as previously outlined; $B_{F O F D M}=B_{O F D M} / 2$. We set $B_{O F D M}=f_{c} /[0.1: 0.1: 1]$, i.e. $B_{O F D M}=$ $[6.0,3.0,2.0,1.5,1.2,1,0.85,0.75,0.667,0.6] \mathrm{MHz}$, following the same method employed in our previous work [18]. Testing this series of signal bandwidths will provide substantial insight into the performance of high capacity links that are limited by the PLED bandwidth, particularly considering that the spectral efficiencies between the two formats are maintained and FOFDM has half the bandwidth requirement.

In the next section, we investigate the bit error rate (BER) in a bit-by-bit manner as a function of $E_{b} / N_{0}$. We also measure the electrical power penalty experienced by each modulation format under test by comparing the measured $E_{b} / N_{0}$ against the theoretical value for the given modulation format at BER values of $10^{-6}$ and $10^{-3}$. The first BER target, $10^{-6}$, was selected in agreement with the International Telecommunications Union (ITU) standard for uncoded links ITU-T G.826 [28], while the $10^{-3}$ target was selected due to its close proximity to the BER threshold for a number of error correcting channel codes commonly used in optical systems. As a result of these considerations, at least $10^{7}$ bits were transmitted in each case in order to ensure the statistical validity of this work with the resulting power penalties are presented for these two BER targets. Finally, we measure the PAPR across the range of $N$.

\section{RESULTS}

The results will be discussed as a comparison between FOFDM and OFDM for the selected values of $k$ and $k_{o}$, starting with the BER performance of the link under test, and then with the power penalties. 


\section{A. BER Performance}

In Fig. 5(a), the BER curves for 2-PAM F-OFDM are shown only for $f_{c} / B_{O F D M}=[0.1,0.2,0.3,0.5]$ for values of $N$ ranging from $N=16 \longrightarrow 1024$. Other values were tested but not shown for visual clarity, especially considering no improvement is noticed for $f_{c} / B_{O F D M}>0.5$. The most important feature of Fig. 5(a) is that the $10^{-6}$ target was reached and no error floor was observed for every single signal bandwidth tested. For $f_{c} / B_{O F D M}=0.1$, the OFDM (FOFDM) signal $\mathrm{BW}$ is 6 (3) $\mathrm{MHz}$ and the $E_{b} / N_{0}$ required to achieve a BER of $10^{-6}$ ranges from $\sim 19.5 \mathrm{~dB}$ for $N=16$ down to $\sim 19.5 \mathrm{~dB}$ for $N=1024$, representing a $\sim 1.5 \mathrm{~dB}$ difference, and a dependence on $N$. This dependence on $N$ is consistent throughout the results (as can be observed by inspection of Fig. 5(a)-(d)) and the variation in $E_{b} / N_{0}$ decreases with increasing $f_{c} / B_{O F D M}$. This dependence is due to the fact that for a smaller value of $N$, wider subcarrier bandwidths are encountered and such leads to the higher frequency subcarriers suffering higher levels of attenuation per subcarrier (due to the non-flat attenuation profile of the PLED). This effect has been observed in the literature, particularly in band-limited VLC systems [19]. As $N$ increases, the subcarrier bandwidths more closely approximate to flat-bands and hence the $E_{b} / N_{0}$ requirement decreases for any given value of $f_{c} / B_{O F D M}$. For a BER target of ${ }^{-3}$, the required $E_{b} / N_{0}$ is $\sim 13.5-14.5 \mathrm{~dB}$.

For $f_{c} / B_{O F D M}=0.2$, the values for $E_{b} / N_{0}$ at BER targets of $10^{-6}$ and $10^{-3}$ are $\sim 14.5-16 \mathrm{~dB}$ and $\sim 10.5-12 \mathrm{~dB}$, respectively, corresponding to $N=1024 \longrightarrow 16$, representing an improvement of $\sim 3.5 \mathrm{~dB}$ over the $f_{c} / B_{O F D M}=0.1$ case for the $10^{-6}$ BER target and a gain of $\sim 2.5-3 \mathrm{~dB}$ for the $10^{-3}$ BER target. For $f_{c} / B_{O F D M}=0.3: 0.5$, the $E_{b} / N_{0}$ values for the $10^{-6}$ BER target are as follows; $\sim\{13-14,12-13,11.5-$ $12\} \mathrm{dB}$. For values of $f_{c} / B_{O F D M}<0.5$, the range remains 11-11.5 dB and the reason for this will be discussed in the next paragraphs. For $10^{-3}$, the respective $E_{b} / N_{0}$ values are: $\sim\{8.5$ $9,7.75-8.25,7.25-7.75,7-7.5\} \mathrm{dB}$. As in the previous case, for $E_{b} / N_{0}$ values less than 0.5 , there is no improvement. This makes sense for FOFDM, since $B_{F O F D M}=B_{O F D M} / 2$ and for $f_{c} / B_{O F D M}=0.5, B_{F O F D M}=f_{c}$ and hence the signal bandwidth is within the modulation bandwidth of the PLED and hence no ISI is induced from this value onwards.

One interesting point to note is that even in the case where $B_{F O F D M}=f_{c}$, the BER profile does not perfectly approach the theoretical $E_{b} / N_{0}$ value of $10.7 \mathrm{~dB}$ for a BER of $10^{-6}$. The reason for this is due to the model of the PLED, which is based on a resistor-capacitor first order low-pass filter as reflected in eqn. (4) and therefore it is a sub-optimum filter that does not have the advantage of a matched filter. Generally, for $f_{c} / B<1$, the noise power is dominated by the ISI, which increases expectedly with increasing $f_{c} / B$, as will be seen in the next paragraphs. For $f_{c} / B=1$, the power penalty reaches a minimum, which is observed to be $\sim 1 \mathrm{~dB}$. For $f_{c} / B>1$, the power penalty also also increases as expected due to the additional noise power introduced by the excess modulation bandwidth in comparison to the signal bandwidth.

For the OFDM system under the same conditions; i.e.
$N=2^{/ 4: 10 /}, k_{o}=2$, approximately equivalent performance was observed, as is illustrated in Fig. 5(b). For a BER target of $10^{-6}$, the measured $E_{b} / N_{0}$ values for $f_{c} / B_{O F D M}=0.1: 1$ were as follows: $\sim\{21-22.75,18-19,16-16.75,14.75-15.5$, 13.5-14.5, 13-13.5, 12.5-13.5, 11.5-12\} dB, all of which exceed the respective F-OFDM cases with the equivalent spectral efficiency. One observation is that when the band-limitations are removed, i.e. when $f_{c} / B_{O F D M}=0.5$ in the FOFDM case and $B_{O F D M} / f c=1$ in the OFDM case, the required $E b / N_{0}$ values are equivalent. This closely matches the original proposal of F-OFDM in [10] (and experimental verification in [16]) which showed identical performance of the two formats when no ISI or band-limitations/filtering were assumed. For the $10^{-3}$ BER target, the $E_{b} / N_{0}$ values are adjusted to: $\{16.5-$ $18,13-14,11.5-12,10-10.5,9-9.5,8.5-9,8-8.5,7.75-8,7.5-$ 8 , and $7.5-8\} \mathrm{dB}$. These values follow the same trend as previous, where the F-OFDM are all smaller than their OFDM counterparts, until the band-limitation conditions are removed. This trend in comparative BER performance (i.e. F-OFDM outperforms OFDM by several dB) under the same spectral efficiency and conditions outlined in this paper, extends to any error target, generally, where an error floor is not met.

It remains to be seen whether the same is true for higher orders of $k$ and hence, we repeat the test for $k=2$ and $k_{o}=4$. The BER curves for these conditions are shown in Fig. 5(c) and (d) for F-OFDM and OFDM, respectively. In Fig. 5(c), only $f_{c} / B_{O F D M}=0.1$ and 0.5 are shown, since these are the most and least band-limited conditions, respectively, for FOFDM. Clearly, for $f_{c} / B_{O F D M}=0.1$ and $N=16$, the system reaches a BER floor of $\sim 10^{-4}$. This is attributed to the larger subcarrier bandwidth mentioned earlier and the higher levels of attenuation experienced by each subcarrier. For $N=$ 32 , the BER target is reached when $E_{b} / N_{0} 25.5 \mathrm{~dB}$, however it should be noted that an error floor is reached for BER values below $10^{-6}$. For the remaining values of $N$, the BER target is met when $E_{b} / N_{0}=22--23 \mathrm{~dB}$. For $f_{c} / B_{O F D M}=$ $0.1: 0.5$, the $E_{b} / N_{0}$ values are as follows for all $N$ except $N=16: \sim\{19-21,17-18.5,16-17.5,15.5-16.5,15-16\} \mathrm{dB}$. In a similar manner as the study, the $E_{b} / N_{0}$ values do not improve further for the remaining $f_{c} / B_{O F D M}$.

For the $10^{-3}$ BER target, the following values were measured: $\sim\{17-21,13.75-16,12.25-14,11.5-13,11-12.5\} \mathrm{dB}$ for $f_{c} / B_{O F D M}=0.1$ up to 0.5 and $11-12.5 \mathrm{~dB}$ beyond. For OFDM, the same trend occurs, as can be inferred from Fig.5(d). Interestingly, several additional error floors emerge for OFDM, which are attributed to the wider subcarrier bandwidths as previously mentioned. The individual $E_{b} / N_{0}$ values are not listed here, however they can be clearly seen to follow the same trend.

\section{B. Power Penalties}

Further insight can be gained by analysis of the electrical power penalty for the signals under test, which are illustrated in Fig. 6(a) for F-OFDM and OFDM using 2-PAM and 4QAM, respectively for the $10^{-6}$ BER target (the $10^{-3}$ target is shown inset with the same axes ranges). It is therefore clear that the power penalty (in $\mathrm{dB}$ ) increases approximately exponentially, with the linear reduction of bandwidth, for both BER 

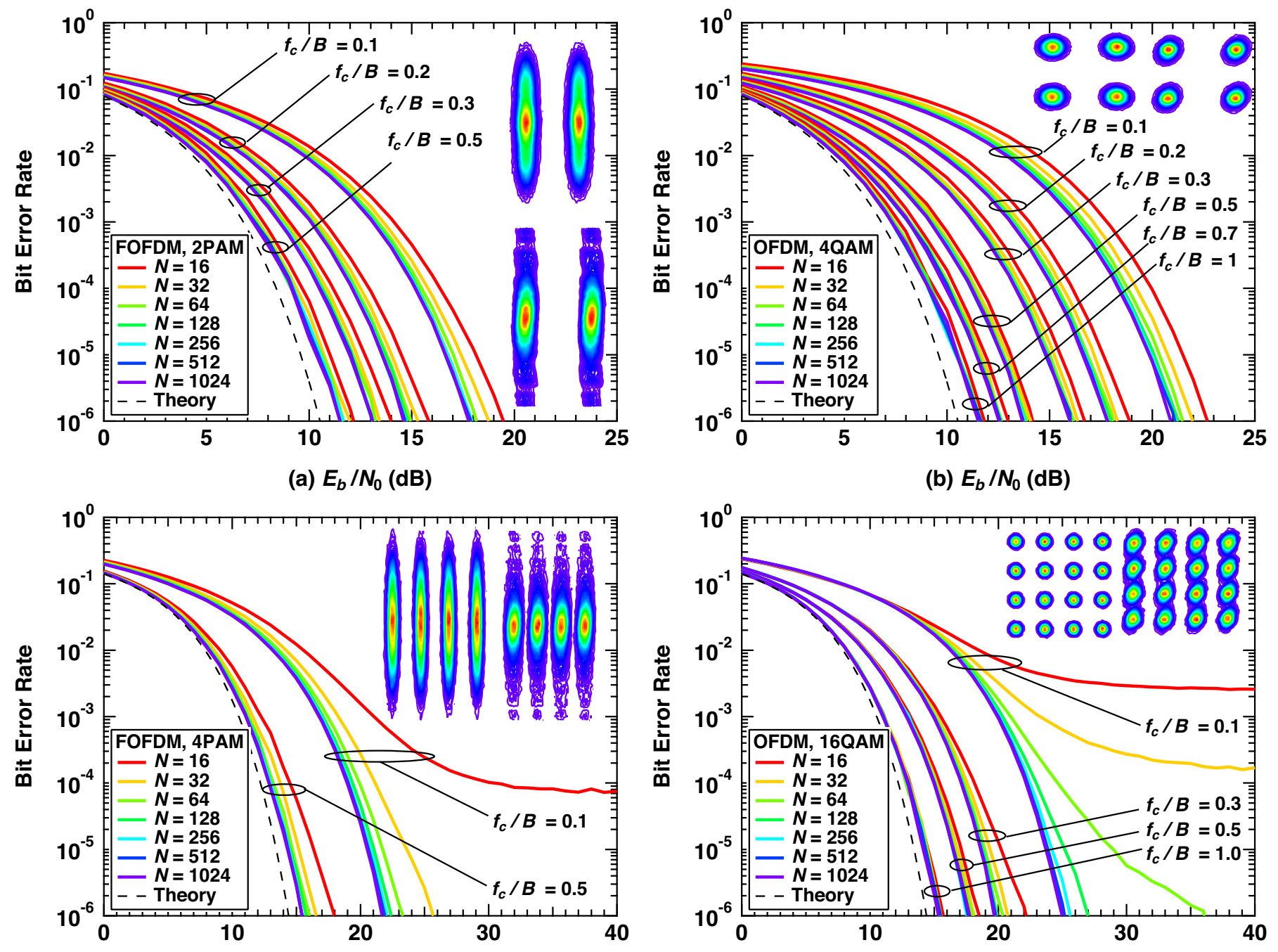

(c) $E_{b} / N_{0}(\mathrm{~dB})$

(d) $E_{b} / N_{0}(\mathrm{~dB})$

Fig. 5. Several of the BER profiles of (a) F-OFDM modulated with 2-PAM, (b) OFDM modulated with 4-QAM, (c) F-OFDM modulated with 4-PAM, and (d) OFDM modulated with 16-QAM. It is clear from inspection of (a) and (b) that the BER profiles of F-OFDM and conventional OFDM under the same band-limitated conditions and spectral efficiency follow the same trend, however, FOFDM offers several dBs lower $E_{b} / N_{0}$ due to the lower signal bandwidth. In the worst case, where $B / f c=0.1$, i.e. the signal bandwidth exceeds the PLED bandwidth by ten times, the $E_{b} / N_{0}$ penalty is $7-8 \mathrm{~dB}$ for FOFDM and 10-12 dB for OFDM in comparison to the theoretical value for 2-PAM/4-QAM, meaning that 18-20 dB are required to support the FOFDM link, while 21-23 dB are required for OFDM. Inset in Fig. 5(a), constellations are shown for FOFDM when $N=16, E_{b} / N_{0}=20$ and $f_{c} / B_{O F D M}=0.1$ (top) and $N=1024, E_{b} / N_{0}=12$ and $f_{c} / B_{O F D M}=1$ (bottom). Both provide error free performance. Interestingly, the loss of orthogonality in the quadrature domain is clear to see, with an abundance of interference present, whereas in the real part, error free performance is maintained. Inset in Fig. 5(b) the constellations are also shown, with the expected shape for 4-QAM (left) where $N=1024, E_{b} / N_{0}=12$ and $f_{c} / B_{O F D M}=1$ and (right) where $N=16, E_{b} / N_{0}=23$ and $f_{c} / B_{O F D M}=0.1$. The same is true for higher orders of $k$ and $k_{o}$ as can be observed in (c) and (d), with approximately equivalent power penalties in comparison to (a) and (b). The equivalent constellations are shown inset, where the conditions are set as follows in both figures: $N=1024, E_{b} / N_{0}=16$ and $f_{c} / B_{O F D M}=1$ (left) and $N=16, E_{b} / N_{0}=30$ and $f_{c} / B_{O F D M}=0.1$ (right)

targets. As the signal bandwidth $B$ increases in comparison to the fixed $600 \mathrm{kHz}$ offered by the PLED, the interference power introduced by the longer ISI span increases. There is an $\sim 3 \mathrm{~dB}$ difference between the two modulation formats while $f_{c} / B_{O F D M}>0.5$. For FOFDM, when $f_{c} / B_{O F D M}<0.5$, the power penalty remains constant at $\sim 1 \mathrm{~dB}$ as expected. The difference between the two modulation formats reduces in this range because the ISI experienced by OFDM gets lower as $f_{c} / B_{O F D M} \longrightarrow 1$. The maximum attenuation experienced at a BER of $10^{-6}$ was $\sim 9$ and $12 \mathrm{~dB}$ when $f_{c} / B_{O F D M}=1$ for FOFDM and OFDM, respectively, corresponding to a signal bandwidth of $6 \mathrm{MHz}$. The literature has reported that high signal-to-noise ratios are present in the majority of VLC links, where values exceed $40 \mathrm{~dB}$ with proper distribution of transmitters [29], and hence, there is sufficient signal power to support this high signal bandwidth link, regardless of the restricted PLED bandwidth. The inset power penalty for the BER target of $10^{-3}$ follows the same pattern. Finally, it is also clear that the lower values of $N$ have higher power penalty due to the aforementioned higher subcarrier bandwidths. It can clearly be seen from Fig. 6(b), which shows the electrical power penalty of FOFDM and OFDM modulated by 4-PAM and 16-QAM respectively, that the performance of each format is approximately equivalent in terms of power penalty to the previous measurements in Fig. 6(a), indicating a clear independence of power penalty from $k$.

In Fig. 7 , the measured $E_{b} / N_{0}$ gain of FOFDM in comparison to OFDM is shown for $k=1$ and $k_{o}=2$ with a 


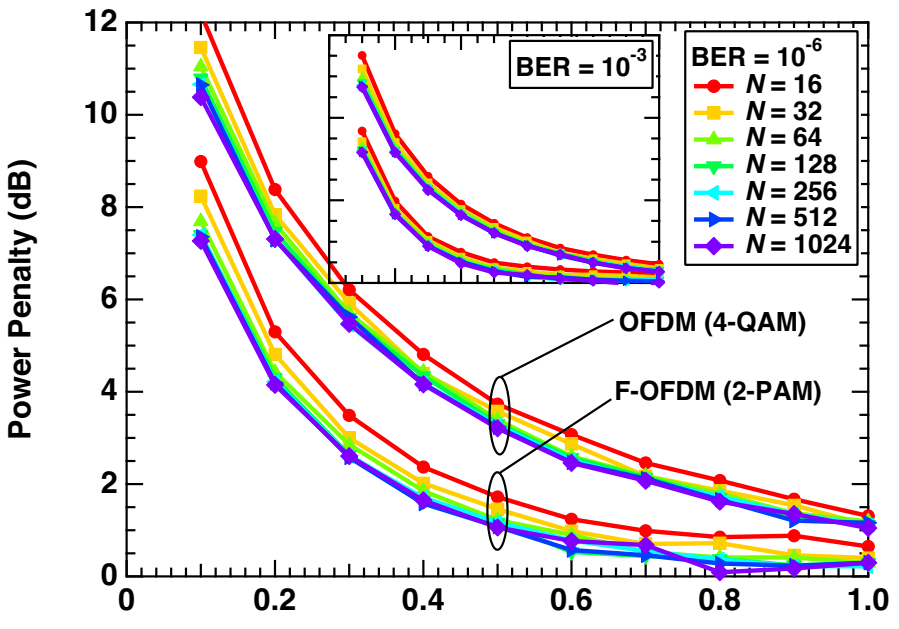

(a) $f_{c} / B$

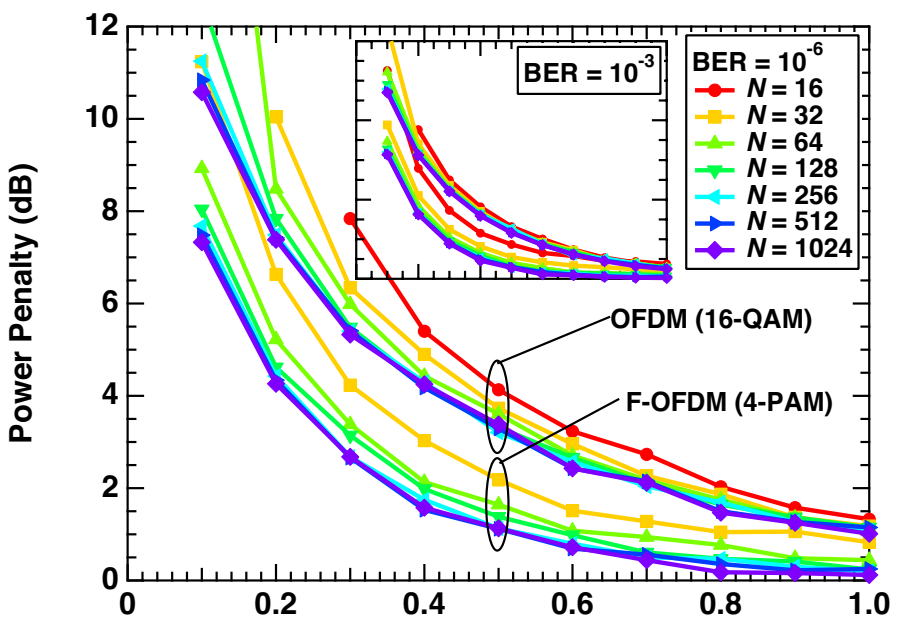

(b) $f_{c} / B$

Fig. 6. The measured electrical power penalties for (a) F-OFDM modulated with 2-PAM and OFDM modulated with 4-QAM for BER targets of $10^{-6}$ (and inset $10^{-3}$ ). The power penalties exhibit an exponential increase as the system becomes increasingly band-limited and both BER targets display approximately the same exponential shape. In (b), the electrical power penalty for F-OFDM modulated with 4-PAM and OFDM modulated with 16-QAM is shown, which both show approximately equivalent performance to that observed in (a).

BER target of $10^{-6}$. Clearly, as $f_{c} / B_{O F D M}$ increases and the system becomes more band-limited, the advantage of FOFDM having half the bandwidth of OFDM starts to dominate and a $3 \mathrm{~dB}$ gain in power penalty can be observed, which appears to be independent of $N$. Further independence from $k$ and $k_{o}$ with the BER target can be observed, following the same trend, but are not shown for clarity considerations.

Finally, in Fig. 8, the PAPR of the signal used to modulate the PLED is shown. We observe that the PAPR for F-OFDM is consistently $\sim 0.5 \mathrm{~dB}$ less in comparison to OFDM across the entire range of subcarriers. The PAPR increases with an increasing order of $N$ because the number of sines/cosines increases and the probability of them aligning and adding by constructive superposition at the output of the IDCT/IFFT increases proportionally. This is also linked to the reason why FOFDM offers lower PAPR than OFDM for any value of $N$, i.e. there is no sine component and as such only the cosines contribute to the signal power. Obviously, a signal

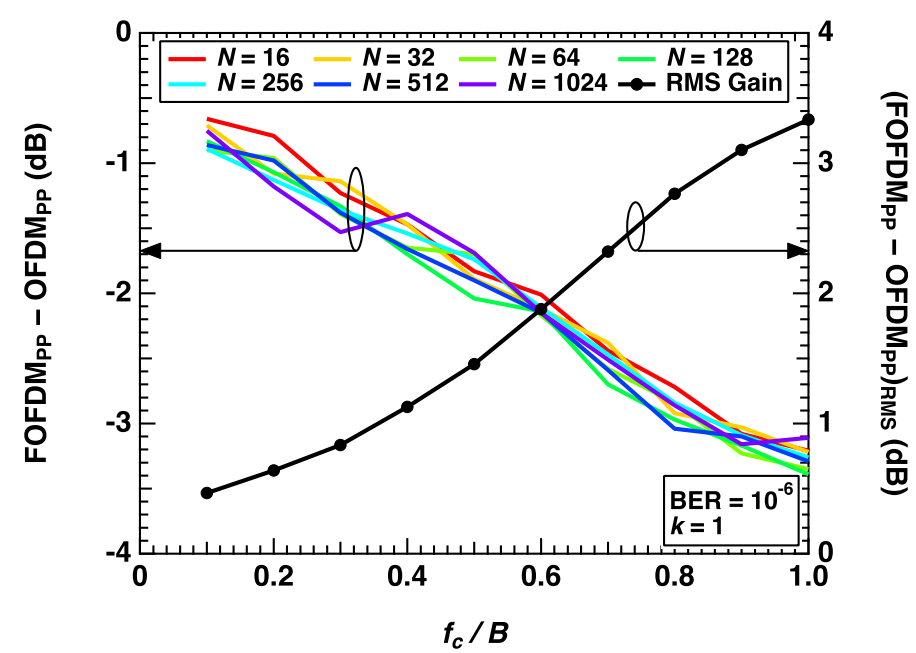

Fig. 7. The net power penalty gain for FOFDM over OFDM for $k=1$ (and $k_{o}=2$ ). The negative index indicates that FOFDM requires less power than OFDM for the same band-limitation conditions. On the right hand side, the root-mean-squared (RMS) gain of the overall system is shown.

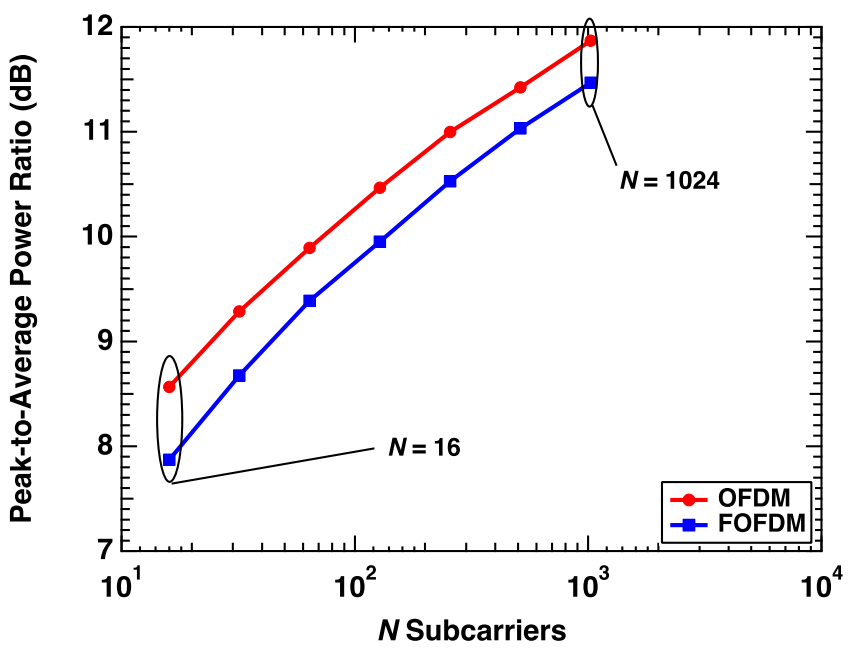

Fig. 8. The measured PAPR for F-OFDM and OFDM with $k=1$ and $k_{o}=2$. The PAPR increases with increasing $N$ as is expected due to the additional number of carriers contributing the the signal.

with lower PAPR is advantageous for communication systems due to limitations caused by transmit devices non-linearity. Lower PAPR is demonstrated for lower values of $N$ and hence, a trade-off emerges. Considering the BER profiles and power penalties are dependent on $N$, using a lower number of subcarriers can be considered advantageous when the nonlinearity of the system is the key limiting factor (i.e. in some conventional VLC systems). However, in a system such as the one discussed in this work, where the bandwidth is the bottleneck and high transmission speeds are required, we argue that using a higher number of subcarriers is desirable and the additional PAPR is a penalty that must be paid, especially when considering the highly linear nature of the PLEDs used.

The results above show two key advantages of FOFDM over OFDM. First, there is approximately 50\% bandwidth saving when the same modulation format is used. Second, when modulation formats of equal spectral efficiencies are used, FOFDM shows a serious BER advantage compared to OFDM in band-limited systems. Hence, we propose FOFDM for further investigation in VLC systems as a viable candidate 
to replace conventional OFDM.

\section{CONCLUSiON}

In this paper we have proposed, for the first time, fastOFDM for highly band-limited VLC links based on organic polymer light-emitting diodes. We have comparatively illustrated the BER performance of FOFDM against conventional OFDM in an increasingly band-limited environment. We show that when the signal bandwidth is ten times greater than the PLED modulation bandwidth, a BER target of $10^{-6}$ can be achieved at the cost of a 7-8 $\mathrm{dB}$ electrical power penalty, depending on $N$, in comparison to the 10-11 dB penalty experienced by OFDM against the theoretical $E_{b} / N_{0}$ value, for any value of $k$. Furthermore, we show that for systems with equivalent spectral efficiency, FOFDM offers approximately $3 \mathrm{~dB}$ net gain for any value of $N$. Due to all of these reasons, we believe F-OFDM is a promising candidate for research in highly band-limited VLC systems.

\section{ACKNOWLEDGMENT}

The authors would like to thank Dr Francesco Bausi, of the National Physics Laboratory, for performing the PLED fabrication and several of the characterisation techniques, and Prof. Franco Cacialli for facilitating the PLED fabricating.

This work was supported by the UK EPSRC grant, EP/P006280/1: Multifunctional Polymer Light-Emitting Diodes with Visible Light Communications (MARVEL).

\section{REFERENCES}

[1] , "Data traffic forecast update 20142019," Cisco Visual Network Index Global Mobile, 2015.

[2] F. M. Wu, C. T. Lin, C. C. Wei, C. W. Chen, Z. Y. Chen, H. T. Huang, and S. Chi, "Performance comparison of OFDM signal and CAP signal over high capacity RGB-LED-based WDM visible light communication," IEEE Photonics Journal, vol. 5, no. 4, pp. $7901507-$ 7901507 , Aug 2013.

[3] J. Wang, Y. Xu, X. Ling, R. Zhang, Z. Ding, and C. Zhao, "PAPR analysis for OFDM visible light communication," Opt. Express, vol. 24, no. 24 , pp. $27457-27474$, Nov 2016.

[4] Y. Wang, X. Huang, L. Tao, J. Shi, and N. Chi, "4.5-Gb/s RGBLED based WDM visible light communication system employing CAP modulation and RLS based adaptive equalization," Opt. Express, vol. 23, no. 10, pp. 13626-13633, May 2015.

[5] H. Aziz, Z. D. Popovic, N.-X. Hu, A.-M. Hor, and G. Xu, "Degradation mechanism of small molecule-based organic light-emitting devices," Science, vol. 283, no. 5409, pp. 1900-1902, 1999.

[6] T. M. Brown and F. Cacialli, "Contact optimization in polymer lightemitting diodes," Journal of Polymer Science Part B: Polymer Physics, vol. 41, no. 21, pp. 2649-2664, 2003.

[7] P. Li, F. Di Stasio, G. Eda, O. Fenwick, S. O. McDonnell, H. L. Anderson, M. Chhowalla, and F. Cacialli, "Luminescent properties of a water-soluble conjugated polymer incorporating graphene-oxide quantum dots," ChemPhysChem, vol. 16, no. 6, pp. 1258-1262, 2015.

[8] I. A. Barlow, T. Kreouzis, and D. G. Lidzey, "High-speed electroluminescence modulation of a conjugated-polymer light emitting diode," Applied Physics Letters, vol. 94, no. 24, p. 243301, Jun. 2009.

[9] P. A. Haigh, F. Bausi, H. L. Minh, I. Papakonstantinou, W. O. Popoola, A. Burton, and F. Cacialli, "Wavelength-multiplexed polymer LEDs: Towards $55 \mathrm{mb} / \mathrm{s}$ organic visible light communications," IEEE Journal on Selected Areas in Communications, vol. 33, no. 9, pp. 1819-1828, Sept 2015.

[10] M. Rodrigues and I. Darwazeh, "Fast OFDM: A proposal for doubling the data rate of OFDM schemes," in ICT, 2002.
[11] X. Ouyang, H. Zhang, Y. Chen, S. Alam, M. N. Petrovich, F. Poletti, D. J. Richardson, F. C. G. Gunning, and J. Zhao, "Experimental demonstration of improved equalization algorithm for IM/DD fast OFDM," IEEE Photonics Technology Letters, vol. 27, no. 16, pp. 1780-1783, Aug 2015.

[12] L. Zhao, J. He, Z. Zhou, R. Deng, and L. Chen, "The research of optical fast OFDM based on channel estimation algorithm," IEEE Photonics Journal, vol. 8, no. 3, pp. 1-5, June 2016.

[13] J. Zhou, Y. Qiao, T. Zhang, E. Sun, M. Guo, Z. Zhang, X. Tang, and F. Xu, "FOFDM based on discrete cosine transform for intensitymodulated and direct-detected systems," Journal of Lightwave Technology, vol. 34, no. 16, pp. 3717-3725, Aug 2016.

[14] W. L. Chin, "Maximization of effective signal power in DCT window for symbol time synchronization in optical fast OFDM," Journal of Lightwave Technology, vol. 31, no. 5, pp. 740-748, March 2013.

[15] Y. Wang, Y. Zhou, T. Gui, K. Zhong, X. Zhou, L. Wang, A. P. T. Lau, C. Lu, and N. Chi, "Efficient MMSE-SQRD-based MIMO decoder for SEFDM-based 2.4-Gb/s-spectrum-compressed WDM VLC system," IEEE Photonics Journal, vol. 8, no. 4, pp. 1-9, Aug 2016.

[16] J. Zhao and A. D. Ellis, "A novel optical fast OFDM with reduced channel spacing equal to half of the symbol rate per carrier," in Optical Fiber Communication Conference. Optical Society of America, 2010, p. OMR1.

[17] F. Xiong, "M-ary amplitude shift keying OFDM system," IEEE Transactions on Communications, vol. 51, no. 10, pp. 1638-1642, 2003.

[18] P. A. Haigh, S. T. Le, S. Zvanovec, Z. Ghassemlooy, P. Luo, T. Xu, P. Chvojka, T. Kanesan, E. Giacoumidis, P. Canyelles-Pericas, H. L. Minh, W. Popoola, S. Rajbhandari, I. Papakonstantinou, and I. Darwazeh, "Multi-band carrier-less amplitude and phase modulation for bandlimited visible light communications systems," IEEE Wireless Communications, vol. 22, no. 2, pp. 46-53, April 2015.

[19] P. A. Haigh, A. Burton, K. Werfli, H. L. Minh, E. Bentley, P. Chvojka, W. O. Popoola, I. Papakonstantinou, and S. Zvanovec, "A multiCAP visible-light communications system with $4.85-\mathrm{b} / \mathrm{s} / \mathrm{Hz}$ spectral efficiency," IEEE Journal on Selected Areas in Communications, vol. 33, no. 9, pp. 1771-1779, Sept 2015.

[20] R. Friend, R. Gymer, A. Holmes, J. Burroughes, R. Marks, C. Taliani, D. Bradley, D. Dos Santos, J. Bredas, M. Lögdlund et al., "Electroluminescence in conjugated polymers," Nature, vol. 397, no. 6715, pp. 121-128, 1999.

[21] N. Johansson, F. Cacialli, K. Xing, G. Beamson, D. Clark, R. Friend, and W. Salaneck, "A study of the ITO-on-PPV interface using photoelectron spectroscopy," Synthetic Metals, vol. 92, no. 3, pp. 207 - 211, 1998.

[22] F. B. Ogunkoya, W. O. Popoola, and S. Sinanovi, "Pilot-assisted PAPR reduction technique for O-OFDM using multiple leds in VLC systems," in 2016 IEEE International Conference on Communications Workshops (ICC), May 2016, pp. 309-314.

[23] P. A. Haigh, Z. Ghassemlooy, I. Papakonstantinou, F. Arca, S. F. Tedde, O. Hayden, and E. Leitgeb, "A 1-Mb/s visible light communications link with low bandwidth organic components," IEEE Photonics Technology Letters, vol. 26, no. 13, pp. 1295-1298, July 2014.

[24] J. M. Kahn and J. R. Barry, "Wireless infrared communications," Proceedings of the IEEE, vol. 85, no. 2, pp. 265-298, Feb 1997.

[25] R. X. G. Ferreira, E. Xie, J. J. D. McKendry, S. Rajbhandari, H. Chun, G. Faulkner, S. Watson, A. E. Kelly, E. Gu, R. V. Penty, I. H. White, D. C. OBrien, and M. D. Dawson, "High bandwidth GaN-based microLEDs for multi-Gb/s visible light communications," IEEE Photonics Technology Letters, vol. 28, no. 19, pp. 2023-2026, Oct 2016.

[26] N. Chi, M. Zhang, Y. Zhou, and J. Zhao, "3.375-Gb/s RGB-LED based WDM visible light communication system employing PAM-8 modulation with phase shifted manchester coding," Opt. Express, vol. 24, no. 19, pp. $21663-21673$, Sep 2016.

[27] D. Tsonev, H. Chun, S. Rajbhandari, J. J. D. McKendry, S. Videv, E. Gu, M. Haji, S. Watson, A. E. Kelly, G. Faulkner, M. D. Dawson, H. Haas, and D. O'Brien, "A 3-Gb/s single-LED OFDM-based wireless VLC link using a gallium nitride $\mu$ LED," IEEE Photonics Technology Letters, vol. 26, no. 7, pp. 637-640, April 2014.

[28] I.-T. R. ITU-T G, "826, error performance parameters and objectives for international. constant bit rate digital paths at or above the primary rate," ITU-T tech. rep. Genebra, Suiça, 1999.

[29] I. Stefan, H. Burchardt, and H. Haas, "Area spectral efficiency performance comparison between VLC and RF femtocell networks," in 2013 IEEE International Conference on Communications (ICC), June 2013, pp. 3825-3829. 